\title{
Estándares de desempeño docente, ¿llegaron para no quedarse?
}

\author{
Claudia Celina Gaytán Díaz \\ Irma Mercedes Cano Medrano \\ María Araceli Gutiérrez Reyes \\ Centro de Investigación y Docencia
}

\section{Resumen}

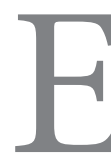

1 profesorado de la escuela primaria atraviesa por un momento histórico: se le presentan distintos planteamientos curriculares que demandan cambios en sus prácticas pedagógicas, lo que conlleva a insertarse en procesos de formación continua con los que se pretende mejorar su desempeño en el aula, que será evaluado a través de distintos mecanismos. En este artículo se describen los resultados de una investigación que tiene el objetivo de identificar las Representaciones Sociales (RS) que el profesorado de educación primaria ha construido sobre la propuesta de evaluación basada en Estándares de Desempeño Docente, partiendo de una perspectiva teórica ubicada en la psicología social a la que se adscribe la teoría de las RS desarrollada por Serge Moscovici y utilizando un diseño metodológico fundamentado en los estudios de la Escuela de Ginebra, cuyo máximo exponente es Wi- llen Doise. Se diseñó y aplicó un cuestionario que integra dos técnicas de recolección de datos: asociación libre de palabras y escala de valoración semántica. Dentro de los principales resultados fue posible identificar una gran dispersión en cuanto al diccionario de palabras. A partir de la contextualización en el ámbito educativo, se definieron cuatro categorías que conforman el campo de representación: a) Niveles de medición; b) Formación docente; c) Objetivos planteados y, d) Inapropiados y, a través del análisis de resultados de la Escala de Valoración Semántica, se identificó un porcentaje considerable que manifiesta que los Estándares de Desempeño Docente son: ineficaces, desconocidos, ambiguos, inadecuados e incongruentes con su práctica docente.

Palabras clave: representaciones sociales, estándares de desempeño docente, educación primaria, docentes. 
REVISTA DE INVESTIGACIÓN EDUCATIVA DE LA REDIECH N. 7 ISSN: 2007-4336

\section{Introducción}

Aboites (2012a) menciona que "a partir de 1990 con la llegada de la evaluación moderna cambia en México radicalmente la concepción y práctica de lo que era antes una actividad académica principalmente a cargo de los maestros, escuelas y universidades" (p. 14).

Normativamente, a través de la Alianza por la Calidad de la Educación (ACE), suscrita entre el Gobierno Federal y el Sindicato Nacional de Trabajadores de la Educación (SNTE) en el Eje V "Evaluar para Mejorar", se estableció el objetivo de que la evaluación sirva para elevar la calidad educativa, favorecer la transparencia y rendición de cuentas, así como propiciar el diseño adecuado de políticas educativas (SEP, 2011: 1).

A partir de entonces, se han buscado mecanismos que den cuenta del desempeño de alumnos, profesores e instituciones escolares. Concretamente, como propuesta complementaria para evaluar el desempeño del profesorado en educación básica (ya que únicamente se tomaban en cuenta los resultados académicos del alumnado en pruebas estandarizadas) se desarrollaron los Estándares de Desempeño Docente:

...en colaboración con la Organización de Estados Iberoamericanos para la Educación, la Ciencia y la Cultura y el Centro de Estudios Educativos, buscando orientar de manera precisa e informada la transformación de las prácticas de los docentes de Educación Básica, lo que implica un proceso sistemático que contribuirá, de manera significativa, a establecer una cultura de evaluación para la mejora continua (SEP, 2011:21).
De acuerdo con Carlos (2013:225) los Estándares de Desempeño Docente "describen lo que los docentes deben saber y saber hacer; es decir, lo que deben aprender y cómo deben desempeñarse" .

Pero, ¿Cómo recibieron esta nueva propuesta de evaluación las y los profesores de primaria? ¿De qué manera ha permeado el discurso normativo sobre este planteamiento de evaluación en la escuela?, concretamente, ¿Cuáles son las Representaciones Sociales que el profesorado de primaria ha construido sobre los Estándares de Desempeño Docente?

\section{Análisis de fundamentos}

Para Moscovici (1986:472), las representaciones buscan determinar los cambios del pensamiento de la sociedad actual, cómo la novedad y la innovación se convierten en parte de la vida social, además indica que:

En tanto que fenómenos, las RS se presentan bajo formas variadas, más o menos complejas, imágenes que condensan un conjunto de significados; sistemas de referencia que nos permiten interpretar lo que nos sucede, e incluso, dar un sentido a lo inesperado, categorías que sirven para clasificar circunstancias, fenómenos $\mathrm{e}$ individuos con quienes tenemos algo que ver; teorías que permiten establecer hechos sobre ellos: $y$, a menudo, cuando se les comprende dentro de la realidad concreta de nuestra vida social, las representaciones sociales son todo ello junto.

De esta manera, la teoría de las RS, en el campo de la psicología social, "se distancia no solo del enfoque sociológico planteado por Durkheim, sino también de las explicaciones psicológicas basadas en el 
conductismo, y más recientemente en el cognitivismo, y a través de ellas, del individualismo" (Alvarado et al, 2008:43). El estudio de las RS implica el reconocimiento tanto de aspectos psicológicos (cognitivos) de los sujetos como aspectos sociológicos (de pertenencia a grupos).

$\mathrm{Al}$ considerar dichos aspectos, se reconoce que las y los docentes de primaria tienen una historia de vida personal, además una formación inicial específica, se han integrado a comunidades escolares en las que ejercen su profesión, en las aulas han experimentado un sinnúmero de situaciones, enmarcadas dentro de contextos socioeducativos muchas veces adversos; como grupo, han sido blanco de innumerables críticas y expuestos a la opinión pública a través de los medios de comunicación debido a los "pobres" resultados del alumnado en pruebas estandarizadas (ENLACE en educación primaria) que ha sido uno de los ejes rectores de la política educativa propuesta por la Organización para la Cooperación y el Desarrollo Económico (oCDE, 2010) y que ha recibido innumerables críticas, que, por cierto, no se dan a conocer a través de medios masivos de comunicación, debido a que presenta "una visión exageradamente funcional instrumental, lógico formal, contable, administrativa, gerencial" (Moreno, 2012:31).

Dentro de esa misma lógica, se enmarca el planteamiento de los Estándares de Desempeño Docente, para recuperar los elementos que permitan conocer la diferencia entre las actuaciones docentes llevadas a cabo hasta ahora con las que se espera que se desempeñen a partir de la implementación de la RIEB.
Los cambios esperados están en función de las sugerencias que emite la OCDE, y en función de los lineamientos prescritos oficialmente para evaluar la actuación del profesorado en las aulas, aunque Moreno (Ibíd.) opina que es "al margen de consideraciones de diversidad y desigualdad económica, social y cultural" (33); se pretende que se acepte como nueva propuesta y, en función de ello, se actúe en consecuencia, esto puede estar alejado de lo que los actores educativos construyen sobre la misma, es decir, sus RS, que "nos permiten entender la comprensión que el público tiene de la ciencia" (Farr, 2003:158) lo cual implica una nueva teoría, propuesta, modelo o idea.

\section{Procedimiento metodológico}

De acuerdo con Pereira de Sá (citado por Araya, 2002) una línea de investigación para el estudio de las RS (a la que se adscribe el presente trabajo) es la Escuela de Ginebra (sociológica) cuyo máximo exponente es Willen Doise, que se centra en las condiciones de producción y circulación de las RS. Para Doise (citado por Flores, 2005:14):

...la significación de la representación está determinada doblemente por efectos de contexto: en primer lugar por el contexto discursivo a través del cual será formulada o descubierta una RS, en segundo lugar por el contexto sociocultural, es decir, por una parte el contexto ideológico y por la otra, el lugar que ocupa el individuo o el grupo en el sistema social.

Las RS nos permiten construir una perspectiva para situarnos como seres individuales y sociales en el mundo, comprender y explicar los objetos y fenómenos y, en 
consecuencia, justificar nuestras acciones; están integradas por tres dimensiones: el campo de representación, la actitud y la información.

El campo de representación está conformado por los elementos que actúan en una RS como lo son: creencias, valores, vivencias, imágenes y opiniones que se organizan en un todo mediante el proceso de objetivación. Según Araya (2002:40):

La actitud es el aspecto afectivo de las RS ya que es la reacción emocional acerca del objeto o del hecho. Puede ser positiva o negativa, favorable o desfavorable. La información está integrada por todos los conocimientos que se tienen acerca del objeto o del hecho social. La información delata en la actitud según haya sido la cantidad o la calidad de ésta. Aunque tenemos que tener en cuenta que los diferentes grupos sociales mediatizan la cantidad y la precisión, además de que también debemos considerar el origen, pues es muy diferente si se obtiene de un contacto directo con el objeto o por medio de la comunicación social.

En síntesis, conocer o establecer una RS implica determinar qué se sabe (información), qué se cree, cómo se interpreta (campo de la representación) y que se hace o cómo se actúa (actitud) (Ibíd:36).

Partiendo de lo anterior y con el objetivo de identificar las dimensiones de las RS, se integraron dos técnicas de recolección de datos: a: asociación libre de palabras (a través del término inductor Estándares de Desempeño Docente), ya que "favorece la espontaneidad y naturalización de la situación de intercambio, al tiempo que permiten reducir en gran medida la apropiación por los sujetos de los contenidos representacionales de que es portador el investigador" (Banchs, 2000:15) y, b: escala de valoración semántica, porque "los repertorios lingüísticos o universos semánticos producidos por los sujetos contienen aspectos cognitivos, simbólicos y afectivos, dan sentido y direccionalidad a la representación y son los elementos que permiten construir una representación” (Banchs, 2000:15).

La versión final del instrumento se aplicó a una muestra estratificada de 422 docentes de la ciudad de Chihuahua. La captura y análisis de los datos se realizó en el Statistical Packageforthe Social Sciencies (SPSS) con apoyo del programa computacional Excel, a través de generación de tablas (diccionarios de palabras, campos de representación) y gráficos.

\section{Resultados}

De acuerdo con Doise (2005:34) "para delimitar el universo semántico de una RS, puede ser útil obtener información mediante una técnica de asociación de palabras, con términos que se sabe que se utilizan para asociar una imagen al objeto de la representación".

De esta manera a través de la técnica de asociación libre de palabras se incluyó el término inductor Estándares de Desempeño Docente y se obtuvo el diccionario de palabras que se presenta a continuación: 
ESTÁNDARES DE DESEMPEÑo DOCENTE, ¿LLEGARON PARA NO QUEDARSE?

\begin{tabular}{|c|c|c|}
\hline Términos asociados a Estándares de Desempeño Docente & Frecuencia & Porcentaje \\
\hline Profesionalización & 11 & $3.0 \%$ \\
\hline Regulación & 14 & $3.9 \%$ \\
\hline Logros & 14 & $3.9 \%$ \\
\hline Indicadores & 15 & $4.2 \%$ \\
\hline Competencias & 16 & $4.4 \%$ \\
\hline Calidad & 16 & $4.4 \%$ \\
\hline Metas & 16 & $4.4 \%$ \\
\hline Medición & 19 & $5.3 \%$ \\
\hline Capacidad docente & 20 & $5.5 \%$ \\
\hline Escuela & 22 & $6.1 \%$ \\
\hline Mejora & 24 & $6.6 \%$ \\
\hline Perfil & 25 & $6.9 \%$ \\
\hline Nivel de desempeño & 25 & $6.9 \%$ \\
\hline Formación continua & 26 & $7.2 \%$ \\
\hline Inadecuados & 30 & $8.3 \%$ \\
\hline Ideal & 32 & $8.9 \%$ \\
\hline Evaluación & 36 & $10.0 \%$ \\
\hline Totales & 361 & $100.0 \%$ \\
\hline
\end{tabular}

Tabla 1.

Diccionario de palabras que el profesorado de primaria asocia con Estándares de Desempeño Docente

Entre los términos con mayor número de frecuencias, se puede identificar que uno de cada diez profesores asocia los Estándares de Desempeño Docente con la Evaluación, mientras que el $8.9 \%$ lo asocia con un Ideal, el $8.3 \%$ considera que son Inapropiados y el $7.2 \%$ con la Formación Continua, el 6.9\% hace alusión al Nivel de Desempeño, también el 6.9\% menciona el Perfil (docente), la Mejora, con el 6.6\% de menciones y Escuela con el 6.1\%.

Después de identificar el diccionario de palabras que el profesorado asocia al término inductor, se procedió al análisis de los conceptos, se realizó la organización de los mismos de acuerdo a lo que engloba el campo de representación (lo que las personas creen y lo que interpretan) que constituye la contextualización en el ámbito educativo y se determinó una definición de las categorías, posteriormente se integraron las frecuencias en cada una de ellas y se realizó la distribución porcentual, para determinar la proporción específica del campo de representación que las y los docentes han construido sobre el término inductor analizado, que está integrado por cuatro categorías: a) Niveles de Medición, b) Formación Docente, c) Objetivos planteados y, d) Inapropiados, cuyas proporciones se detallan a continuación: 
REVISTA DE INVESTIGACIÓN EDUCATIVA DE LA REDIECH N. 7 ISSN: 2007-4336

\begin{tabular}{|l|c|}
\hline \multicolumn{1}{|c|}{ Categorías } & Porcentaje \\
\hline Niveles de medición & $38.7 \%$ \\
\hline Formación docente & $27.0 \%$ \\
\hline Objetivos planteados & $26.0 \%$ \\
\hline Inapropiados & $8.3 \%$ \\
\hline
\end{tabular}

Tabla 2.

Campo de representación sobre

Estándares de Desempeño Docente

En la primera categoría, el profesorado hace referencia a Niveles de Medición, con el $38.7 \%$ de menciones. A través de términos como Indicadores, Medición, Metas, Logros, Evaluación, Niveles de Desempeño y Regulación, el profesorado indica que los Estándares de Desempeño Docente se asocian con medidas, esto es con evaluaciones de tipo numérico que los posicionan en algún nivel acorde a la consecución de resultados previamente establecidos. Esto, de alguna manera, contraviene lo que propone la OEI (2010:16):

Los estándares de desempeño docente en el aula son un instrumento para la reflexión, el diálogo y el intercambio. Aunque valoran una acción, no pretenden ser una herramienta para elaborar clasificaciones (ranking) de maestros o escuelas, ni mucho menos promover sanciones. La reflexión con base en parámetros es un mecanismo efectivo para la mejora de la práctica docente. Por ello, se construyeron referentes que se basan en una configuración de la profesión docente y se ha pensado en su uso como parámetros, de tal manera que se involucre a los pares y a diversos actores educativos.
La segunda categoría se denomina Formación Docente e integra el $27 \%$ de menciones a las que el profesorado hizo alusión a partir de términos como Profesionalización, Competencias, Capacidad docente, Perfil y Formación Continua, como elementos o aspectos que el profesorado debe desarrollar para desempeñar su práctica. De esta manera, se está de acuerdo con la idea: "el colocar en el centro el desarrollo de competencias para la vida trae como consecuencia la necesidad de transformar la forma de enseñar" (OEI, 2010:17).

La tercera categoría titulada Objetivos planteados, está conformada por el $26 \%$ de menciones a través de las cuales el profesorado asocia los Estándares de Desempeño Docente con términos como Calidad y $\mathrm{Me}$ jora, que tienen que ver con lo que se pretendía alcanzar, desde lo normativo (OEI, 2010:9) a través de su implementación:

Los estándares de desempeño docente tienen como propósito [acompañar al docente] en un proceso de reflexión y autoevaluación, con base en elementos [referentes] comunes sobre algunos asuntos centrales de su práctica. Algunos de los objetivos son: motivar procesos individuales y colectivos de reflexión y evaluación, la identificación de necesidades reales y comunes de formación y sobre todo, el establecimiento de procesos de mejora continua de la práctica docente y de la escuela $(\mathrm{s} / \mathrm{p})$. Además se asocia la evaluación a procesos de mejora continua del desempeño docente, por lo que la evaluación tiene la posibilidad de ser permanente y significativa para la mejora profesional de los maestros, incluso de manera independiente a procesos de contratación, promoción o de estímulos. 
Finalmente, la cuarta categoría se integra únicamente por el término Inapropiados, a través del cual, el $8.3 \%$ del profesorado indica que los Estándares de Desempeño Docente no están acordes con una evaluación adecuada de su práctica; lo cual denota cierto grado de descontento y/o rechazo hacia el establecimiento de esta forma de evaluación de su desempeño profesional. Aboites (2012 b:18) aborda esta temática e indica que:

Medir a una nación pluricultural y compleja, con instrumentos burocráticos únicos y, como las pruebas, discriminatorias en términos de género, socialmente sesgadas y de muy limitado alcance, significa reducir drásticamente el sentido de pertenencia, de identidad, de proyecto futuro (...) reduce lo complejo -de un individuo, de una institución o una nación- a un número abstracto.

Una vez identificado el campo de representación se prosiguió a identificar las actitudes (otra dimensión de las RS) que las y los docentes tienen con respecto a la Rieb en general, cabe destacar que es un acercamiento inicial, puesto que habrá que identificar las actitudes con respecto a los núcleos figurativos que conforman el campo de RS general. Para ello, se realizó el análisis de un ítem de la escala de valoración semántica que fue contestado por el profesorado.

Esta escala presentó a las y los maestros cinco pares de palabras opuestas a partir de la aseveración inicial que indicaba: los estándares de evaluación del desempeño docente son: ineficaces/eficaces, desconocidos/ conocidos, ambiguos/claros, inadecuados/ adecuados $y$, acordes con mi práctica/no acordes con mi práctica, con una escala del 1 al 7, donde los tres primeros valores corresponden a una posición negativa y los tres últimos con una posición positiva. Los resultados se presentan a continuación:

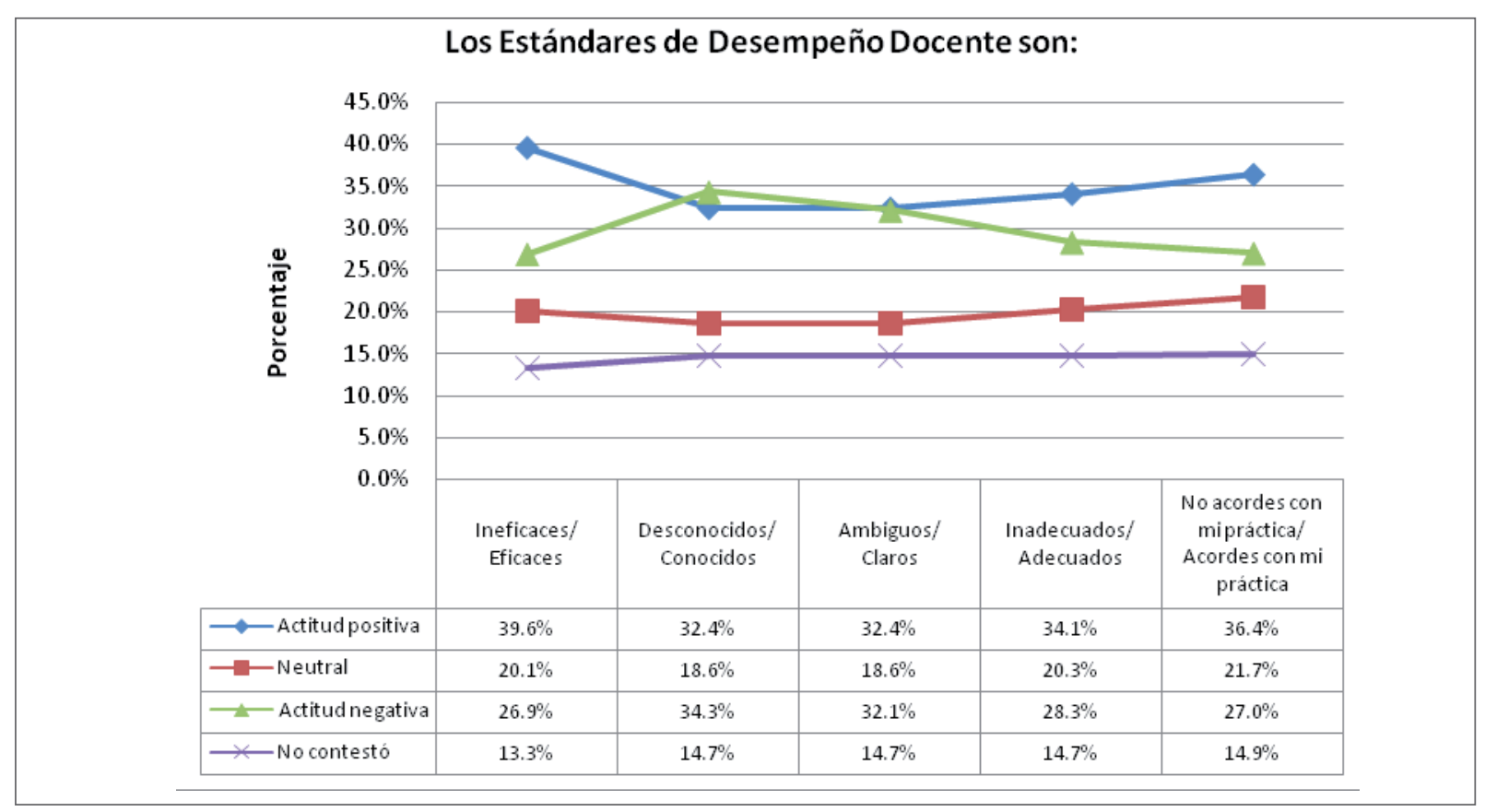

Gráfica 1.

Actitudes manifestadas por el profesorado con respecto a los Estándares de Desempeño Docente OCTUBRE 2013 -MARZO 2014 
Se puede observar que el profesorado que manifiesta una actitud positiva (con respecto al par de palabras) sobre los Estándares de Desempeño Docente no alcanzan el $40 \%$, mientras que, entre el 18.6 y $21.7 \%$ mantienen una posición neutral, entre el 26.9 y $34.3 \%$ manifiesta una actitud negativa y entre el 13.3 y $14.9 \%$ no contestó. Entre lo que se puede rescatar, está el hecho de que casi el $40 \%$ del profesorado manifiesta que los Estándares de Desempeño Docente son "eficaces", sin embargo, el 34.3\% no los conoce.

\section{Conclusiones}

El diccionario de palabras identificado sobre los Estándares de Desempeño Docente como forma de evaluación del profesorado es muy amplio; esta dispersión es un indicador de poca claridad del término para los docentes y es lógico porque hasta la fecha dichos estándares se han quedado solo en una promesa. Al contextualizar dichos términos e identificar el campo de representación, es posible observar que, un sector importante del profesorado (38.7\%) ven en ellos una medición, lo que dista de una evaluación que rescate realmente su desempeño profesional; otro grupo (27\%) resalta la importancia de la formación docente como medio para alcanzarlos y, de esta manera obtener mejores resultados en las evaluaciones de su desempeño; un sector más (26\%), se ha apropiado del discurso normativo al hacer mención de la calidad y la mejora, que han sido algunos de los principales términos que abanderan la reforma educativa; un grupo (8.3\%) que es el más reducido, los considera inapropiados.
Sin embargo, este último porcentaje se incrementa al indagar sobre aspectos más concretos sobre los Estándares de Desempeño Docente ante los cuales no se obtuvieron altos porcentajes en las actitudes positivas con respecto a la eficacia, conocimiento, claridad, adecuación y congruencia con la práctica docente del profesorado $y$, de esta manera, parece que los resultados obtenidos abonan o se unen a la crítica realizada por algunos académicos y sectores magisteriales, a las propuestas de evaluación estandarizadas, que sostienen que "la resistencia no solo ha obligado a modificar los términos en que originalmente se pensaba implantar el proyecto, sino que ha generado demandas y visiones mucho más amplias y distintas de lo que puede ser la educación y la evaluación en el país" (Aboites, 2012 b:19).

\section{Referencias}

Aboites, H. (2012 a ). La disputa por la evaluación en México: historia y futuro. El cotidiano 176 . nov-dic 2012. pp. 5-17. Recuperado en: www.elcotidianoenlinea.com.mx/pdf/17602.pdf

(2012 b ). La medida de una nación. Los primeros años de la evaluación en México. Historia de poder y resistencia. Ítaca/UAM/CLACSO. México.

Alvarado, S., Botero, P. y Gutiérrez, M. (2008). Representaciones sociales. Una mirada a la teoría Moscoviciana. En P. Botero. Representaciones y ciencias sociales. Una perspectiva epistemológica y metodológica. Espacio editorial. Buenos Aires.

Araya, S. (2002). Las representaciones sociales: ejes teóricos para su discusión. Cuaderno de Ciencias Sociales 127. Recuperado el 11 de octubre de 2012 en: http:// flacso.or.cr/index.php/publicaciones-jb-br-jb-i-labor-editorial-jb-i/cuadernos /336-cuaderno-no-127

Banchs, M. (2000). Aproximaciones procesuales y estructurales al estudio de las representaciones sociales. Paperson social representations. Volumen 9. pp. 3.13.15. Recuperado en: www.psich.lsc.ac.uk/psr/ PSR2000/9.3Banch.pdf 
Carlos, G. (Coord.) (2013). Del currículum al aula. Orientaciones y sugerencias para aplicar la RIEB. Ed. Graó/Colofón. México.

Doise, W., Clemence, A. y Lorenzi, F. (2005). Representaciones sociales y análisis de datos. Antologías Universitarias. México.

Farr, R. (1986). Las representaciones sociales. En S. Moscovici. Psicología social II. Pensamiento y vida social. Psicología social y problemas sociales. Ed. Paidós. Barcelona.

Flores, J. (2005). Representaciones sociales y análisis de datos. Antologías Universitarias. México.

Moreno, P. (2012). La política de la calidad y las competencias: deshumanización, alienación y fragmentación del sujeto educativo. Revista de Investigación Educativa de la REDIECH. Octubre 2011-Marzo 2012. pp. 29-36. Chihuahua. México.

Moscovici, S. (1986). Psicología social II. Pensamiento y vida social. Psicología social y problemas sociales. Barcelona: Paidós.

OCDE (2010).Acuerdo de Cooperación México-OCDE para mejorar la calidad de la educación de las escuelas mexicanas. Mejorar las escuelas: Estrategias para la acción en México. Establecimiento de un marco para la evaluación e incentivos para docentes: Consideraciones para México. Resúmenes ejecutivos. Recuperado en http://www.oecd.org/edu/ school/46216786.pdf

Organización de Estados Iberoamericanos (2010). Estándares para la Educación Básica en México. Documento base. Recuperado el 25 de marzo de 2014 en: http://cee.edu.mx/referentes/4-2.pdf

SEP (2011). Plan de estudios de educación primaria. Secretaría de Educación Pública. Recuperado en: http://formacioncontinua.sep.gob.mx/sites/ReformaIntegral/RIEB Plan de Estudios.php 
